\title{
Countermeasures of Wuhan city circle advanced manufacturing Technology Support_-Take Wuhan Robot Industrial Park as an Example
}

\author{
Dong Liang ${ }^{1}$, Guopei Cai ${ }^{2}$ and Zhongwei Zhang ${ }^{3}$ \\ ${ }^{1}$ Jianghan University Business School, Wuhan \\ ${ }^{2}$ Hubei Institute of Macroeconomics, Wuhan \\ ${ }^{3}$ Jianghan University Business School, Wuhan \\ 1925243770@qq.com 2925243770@qq.com 31039857158@qq.com
}

\begin{abstract}
Keywords: Advanced manufacturing; Advanced manufacturing technology; Industrial development; Wuhan robot; Support policies
\end{abstract}

\begin{abstract}
Advanced manufacturing technology is an important part of advanced manufacturing and is a new form of manufacturing. Its advanced manufacturing mode, technical means and management methods represent an industrial strength and level in a country. As a typical representative and main technical means of information technology and advanced manufacturing technology, robotics has become an emerging high-tech technology developed by all the developed countries in the world and its level of development has become one of the most important symbols to measure the degree of technological development in a country. Wuhan Robot Industrial Park is a major engine for promoting regional development and is the need to promote the development of the manufacturing industry. Therefore, on the basis of studying the supporting policies of advanced manufacturing technology in Wuhan metropolitan area, this paper studies the current situation of the development of robot industry in Wuhan. Based on this, the author puts forward countermeasures from the perspectives of personnel training, Wuhan robotics industry cluster and enterprise innovation ability, in order to be able to provide some help for the development of the robotics industry in Wuhan.
\end{abstract}

\section{Preface}

With the rapid development of the robot industry, robot industrial park, as an important carrier and organization of industrial clusters, has become an important link in the development of China's robot industry. At the same time, the competitiveness of the robot industrial park has gradually become an important manifestation of the development of the robotics industry. In 2006, the government announced the "National Medium and Long-term Scientific and Technological Development Plan (2006-2020)", pointing out that in the future, China will regard intelligent service robots as the development direction and propose to study common basic technologies such as manufacturing process, design method and intelligent control, While increasing capital investment. Therefore the development of robots, robot enhance the competitiveness of enterprises has been raised to the national strategic level. The United States has launched a strategy for rapid development of robotics. Europe released a road map for the study and application of European robots. The Japanese government introduced a strategy for the development of new industries and made the robot industry one of the seven key supporting industries. South Korea will serve robotics positioning one of the four pillar industries for future national development. China also clearly included industrial robots to support the focus, the Ministry of Industry last year officially released on the promotion of industrial robot industry guidance. Shanghai, Anhui, Chongqing,, Guangdong, Tianjin and other places have introduced measures to develop the robot industry, the total scale is hundreds of billions. Robot level has become a measure of a country and region manufacturing level and science and technology an important symbol. As a pilot city of "Made in China 2025" pilot project in Wuhan, Wuhan has comparative advantages in automobile manufacturing and must 
catch the trend to catch up.

\section{Wuhan Robot Industry Development Status}

In recent years, the manufacturing industry is constantly moving toward the trend of automation and intelligence. The industrial robot as one of the ten key development areas "2025 Made in China", is an important indicator of industrial automation and industrial 4.0. Industrial robot is a multi-joint manipulator and multi-degree of freedom in order to achieve the operation, is an important way to improve production efficiency, to a certain extent, ease the pressure on equipment manufacturing costs technology and manpower. In the 2015 World Robot Congress ,the president $\mathrm{Xi}$ Jinping pointed out that with the continuous integration of informatization and industrialization, the Intelligent industry represented by robotics is booming and has become an important symbol of scientific and technological innovation in the era. China will include robotics and intelligent manufacturing in the priority areas of national science and technology innovation. In the "Made in China 2025" promulgated by the state in 2016, it is also very clear that the robot has been listed as one of the key development areas of the country.

\section{Development of Industrial Robots Made Great Progress.}

Wuhan Industrial Robot Research started earlier, with strong R \& D capabilities of industrial robots. The city has made a number of important achievements in the study of the basic theory of industrial robots and has had a significant impact across the country. Huazhong University of Science and Technology in recent ten years, the digital manufacturing technology, advanced electronic manufacturing technology, robotics and intelligent control, the establishment of a complex surface wide-line machining theory, put forward a robotic operation planning space geometry inference method, developed a large blade robot " Measuring - operating - processing "integrated (3M) grinding and polishing system, and used in aerospace, energy and automotive and other fields. Research results repeatedly won the National Science and Technology Progress Award and the National Natural Science Award.

At present, the level of industrial robots in Wuhan $\mathrm{R} \& \mathrm{D}$, manufacturing and integration applications is continuously improving, and has been able to independently or jointly research and develop joint-type robots, omnidirectional moveable control robots and remote in-flight robots Production, and achieved the corresponding independent property rights and key core technologies. For example, Wuhan Huachanda intelligent equipment stock company, Developed "with distributed control of their own car" has been identified as Wuhan brand-name products and major scientific and technological achievements in Hubei Province. In addition, the research and development of the four key components that are indispensable to the development of industrial robots have also made great progress. The four major components are robot controller, servo motor, high speed reducer and end effector, and has initially formed a strong supporting capacity. The rapid development of Wuhan robots has provided greater capacity for the industries in Wuhan and saved more manpower costs.

\section{The scale of Wuhan Robot Industry Continues to Expand.}

Wuhan robot industry is currently in the initial stage of industrialization development, Wuhan robot is the most abundant research and development of industrial robots in Hubei Province, the largest concentration of manufacturing enterprises in the region. At present, the East Lake High-tech Zone in Wuhan City has initially formed a cluster effect of the robot industry. The integration of robots in many industries such as laser, electronics, medical treatment and automobile has achieved remarkable results. Based on the introduction of digestion, absorption and re-innovation, Wuhan robots have notable features. Rectangular Cartesian robots have achieved import substitution in the domestic plastic products and food packaging industries, and have been exported overseas on a large scale.

Wuhan robot industry by virtue of many years of industrial enterprises Central enterprises accumulated advantages and flexible mechanism of private enterprises, a wide range of institutions of higher learning and research institutions in close cooperation, made a number of robotics 
research and development achievements., After continuous cultivation, the robot industry in Wuhan City, supporting the basic improvement of the continuous expansion of industrial scale, the construction of the initial system of industrial chain. At present, the city engaged in robot research and development design, manufacturing and engineering applications and spare parts supporting enterprises have more than 40. Wuhan robot enterprises in recent years the strength of the development is constantly growing. According to data provided by People's Daily in Wuhan City, the robot industry is becoming the main force of industrial production in Wuhan Economic and Technological Development Zone.

\section{Robot Industry Agglomeration Effect Initially Formed.}

The robot industry is an important direction and the main battlefield for the development of advanced manufacturing technology. To plan and develop the robot and intelligent equipment industry is an important way to implement the important policy decisions made by Hubei provincial government in accelerating the industrial transformation and upgrading of Hubei Province. It is also an important step for Wuhan to build an industrial upgrading version Industrial transformation and upgrading of major initiatives. The construction and cultivation of the robot industrial base in Wuhan is of great significance for promoting the pilot projects of agglomeration and development of strategic emerging industries in Hubei Province, expanding the scale of the intelligent equipment industry in Hubei Province, and promoting the industrial transformation and upgrading of the province. Although the development of the robot industry in Wuhan lags behind, it has formed a certain agglomeration effect by virtue of its strategic layout and policy support. By vigorously introducing leading robot enterprises and actively nurturing local enterprises, Wuhan City is gradually building a chain of robots with complete chains, advanced technologies and outstanding advantages, guiding the reasonable docking of production and demand, and accelerating the pace of industrial robot integration and application innovation. The development of the domestic robot industry continues to expand its influence.

In the "2017 China Robot Industry Development Report" pointed out that the development of the robot industry in central China has potential for further development. As a core city in the central region, as early as 2014, Wuhan already built the first industrial robot incubator in the country in the East Lake High-tech Development Zone, mainly through housing subsidies, loan interest, financial services, patent incentives, etc. The development of enterprises and start-up teams promoted the agglomeration effect of industrial robotics in Wuhan East Lake High-tech Zone, Integration of small and medium enterprises in the industrial chain, Improve the robot industry chain in Wuhan, the formation of industrial development together.

\section{Wuhan Robot Industry Support Status Analysis}

At present, the world has entered an era of unprecedented innovation and industrial rejuvenation, and strategic emerging industries will become the leading force in economic and social development. As the state accelerates the implementation of the strategy for the rise of the central region, Wuhan City should take the lead in helping the interests of the East and the West and play a fulcrum role in taking the lead in industrial development and economic development and building an important base of energy and raw materials, modern equipment manufacturing and high-tech industrial bases. Last year, Wuhan, as the pilot city for making 2025 in China, already grabbed the first chance in the national policy. The approval of Wuhan Donghu New Technology Development Zone to establish a national demonstration zone of independent innovation brought opportunities for innovation and development in Wuhan and institutional innovation. To further exert the advantage of intensive technology resources and scientific and technological innovation in East Lake High-tech Development Zone and enhance the competitive strength of independent innovation in the city, it will drive the rapid transformation of Wuhan's robot industry development mode. The construction of robot industrial park can't be separated from the joint efforts of the government, enterprises and research institutes. In the process of industry-university-academia cooperation, the government provides policy support and resources support, and plays a leading 
role. The research institutes provide knowledge resources and play a major supporting role.

\section{Robotic Industry has Become the Main Direction.}

As the pace of industrialization of advanced manufacturing technology continues to accelerate, advanced manufacturing industry has become an important part of high-tech industry in Wuhan. As an important part of advanced manufacturing technology, Wuhan Robot has drawn more and more attention in recent years. Hubei Province developed "on promoting six key industries such as manufacturing equipment to accelerate the development of views," "speed up the province's equipment manufacturing industry development action plan", the all clear to enhance industrial robots, additive manufacturing (3D printing) and other high-end Equipment manufacturing industry competitiveness. Last year, the Commission issued "Accelerate the province's smart manufacturing equipment industry development action plan", clearly put forward the industrial robot industry development path of action, action priorities, objectives and safeguards.

According to the requirements of the provincial government, Wuhan City has actively promoted the development of the robot industry and has done many beneficial work. Wuhan Municipal Party Committee and Municipal Government has always insisted on the development of the robot industry as an important starting point for Wuhan's strategy of innovation and development. As a pioneer in accelerating the scale-up and development of Wuhan's industrial transformation and upgrading and "Wisdom in Wuhan", Wuhan Municipality has taken the lead in building a comprehensive innovation reform pilot zone, a national innovation city, Accelerator at the National Manufacturing Innovation Center. Since the beginning of this year, the "Opinions of Wuhan Municipal People's Government of Wuhan on Accelerating the Innovation and Building a National Innovative City" and the "Wuhan Made 2025" policies and measures have been promulgated, and the Wuhan Municipal Information and Industrialization Integrative Action Plan has been launched. The city Equipment manufacturing industry in the 13th Five-Year Plan and so on, both industrial robots and intelligent manufacturing will be included in the development focus and the main direction of development.

\section{Actively Build a Robot Industrial Park as a Carrier to Undertake the Robot Business.}

With the continuous development of our economy and the continuous improvement of the information technology, the demand of the robot market in our country keeps growing. As the intelligent robot industrial park has the advantages of high technology content, broad development prospect and strong industry-driven capability, the local governments have the preparation and planning of industrial robot industry base, fight to win a new round of opportunities in the new wave of industrial automation. May 2016, the leaders of Wuhan City instructed to require "Construction Robot Industrial Park", in September also ordered the actual robot Industrial Park. Wuhan Economic Commission on the basis of full investigation, quickly formed a "Wuhan Robot Industry Research Report" and "Wuhan robot Industrial Park Construction Program" and step up efforts to promote the work

According to the plan, Wuhan Municipal Government will take "a south-north and two-park-driven" mode to Wuhan Robotics Industrial Park. The south park is planned to include 5,700 mu of robot industrial park in East Lake High-tech Zone and a planned area of 5,000 mu in the north park of Caidian Robot Industrial Park. South Park robots are mostly local enterprises, robot incubators have been set up (2 years rent-free), has now settled in nearly 10 enterprises have settled. Robot North Park to the introduction of the main, has started underground gas pipeline relocation, 110,000 volts dedicated substation and other park supporting facilities construction, and actively introduce Jiang new energy vehicles, Guorui intelligent company, Dongfeng painting and a number of industrial robot applications large projects, promoting the digital mode robot project production, Wuhan Ruiming digital factory and other local projects. The park aims to gather more than 200 enterprises by 2020 , forming an annual production capacity of 100,000 industrial robots, 1 million service robots and 1,000 special robots, and realize an annual output value of 50 billion Yuan.

Robotics Industry Supporting Policies Continue to Improve.

In order to attract robot enterprises to enter the Wuhan Robot Industrial Park, Wuhan municipal 
government has also made efforts in various fields in respect of supporting policies on land fees, taxes, government approval and talent introduction. First of all, Wuhan Municipal Government set up government guidance funds, not less than 5 million yuan, used to support the development of robotics enterprises. In terms of land costs, we give more concessions to the production of robots in terms of land prices and provide more favorable conditions during the construction of the project. In terms of tax revenue, the government of Wuhan Municipality prescribes that retained earnings of the robot products produced by the robotic enterprises in Wuhan during the sales process will be repaid in the first five years and returned as part of the special funds for the development of the robot industry. And the city government has been actively encouraging the city robotics enterprises, especially vehicle manufacturers, using robots manufactured by domestic robot manufacturers, according to the amount of the purchase of 5\%-10\% of the government subsidies. In product research and development, Wuhan Municipal Government has set up a Rmb10-30 million subsidy for $\mathrm{R} \& \mathrm{D}$ of enterprises to integrate science and technology resources such as enterprises, governments and universities and establish an R \& D platform for common technologies of open robots. In addition, the municipal government of Wuhan provides financial guarantees for those skilled and marketable robot manufacturers that do need liquidity.

\section{Wuhan Robot Industry Analysis of government Support Measures}

At present, the development of robot industry in Wuhan is in its infancy. Although the industrial scale of Wuhan robot has been expanded under the support of the government in recent years, compared with the robot industrial parks in Shanghai and Changsha, the robot industry in Wuhan is relatively small, There are fewer leading enterprises that can drive the development of the robot industry and the overall competitiveness is weaker. The development of robot industrial park involves a wide range of technical difficulties, its research and development and promotion of the need to invest a lot of manpower, material and financial resources. Looking at developed industrial countries and newly industrialized countries in the development of the robot industry, the government has invested heavily in the local, business, universities and research institutes have also invested considerable power. Therefore, under the circumstance of vigorously developing the robot industry in our country, we should regard promoting the application of the robot industry in manufacturing enterprises as an important task of rejuvenating the city with science and education. The Wuhan Municipal Government should try its best to provide financial support and seize the needs of the industry to overcome Core technology problems, based on institutional innovation, resource integration and reasonable promotion of Wuhan characteristics of the robot industry, we must adhere to the growth of host manufacturers, play a leading role in cultivating leading enterprises and strive to promote the development of robot industry in Wuhan.

\section{Vigorously Foster Wuhan Robot Industry Needs of Personnel Development}

The development of Wuhan robot is inseparable from the personnel training, exchange and introduction of talent, the government in addition to policy support, but also should actively organize various personnel platforms, such as talent exchange platform and the introduction platform, personnel training platform, especially Is a training platform for innovative talents, the use of these platforms for the introduction and training of qualified personnel. Specifically, a special fund for talents can be set up and a green channel can be set up to attract professionals to introduce, train, reward and reward key talents. In the high-end talent plate, focus on key personnel to achieve special training of special personnel. In the field of professionals, we will implement the plan to update the knowledge of skilled personnel, focus on key areas, carry out large-scale continuing education on knowledge renewal and cultivate the professional and technical personnel in urgent need of shortage and backbone. At the grassroots level, we will implement the forging program of highly-skilled professionals in "gold and blue collar" and train the skillful talents urgently needed for industrial restructuring and upgrading.

The talent environment of Wuhan is in the leading position in the whole country, gathering 9 key points such as Robotics Research Center of University of Science and Technology of China, 
Huazhong University of Science and Technology, Wuhan University, Wuhan University of Technology, National CNC System Engineering Research Center and State Key Laboratory of Digital Manufacturing Equipment and Technology Institutions and research institutes. Actively introduce domestic advanced technology innovation team and establish deep cooperation with university research institutes. We will further deepen or retrain the institutions that provide robotics talents, actively promote the implementation of the "Innovative Talents Promotion Plan" in advanced manufacturing sectors, and strengthen the introduction and cultivation of scarce talent in areas such as strategic emerging industries. We should strengthen the cooperation among industry, universities and research institutes in universities, enterprises and research institutes in our country so as to foster a group of young and middle-aged scientific and technological talents, managerial personnel and senior technicians who are young and energetic. In particular, we must cultivate the leading personnel for major equipment research and system design. Actively build a platform for human resources movement both inside and outside of Hubei Province and abroad, establish an information system for overseas students and senior professionals in developed regions, set up an overseas talent pool based on the development of the robot industry in Wuhan, and attract talents back to Wuhan for business start-ups.

\section{Promote the Development of Industrial Clusters Wuhan Robot}

The concept of industrial cluster comes from the conclusion of the experience of western developed countries in regional economy. The meaning of industrial cluster is as follows: It refers to bringing together a group of interrelated companies, suppliers, related industries and specialized institutions and associations in a specific region, This kind of regional agglomeration forms effective market competition, constructs specialized production factors to optimize agglomeration depressions, enables enterprises to share regional public facilities, market environment and external economy, reduces information exchange and logistics costs, and forms regional agglomeration effect, scale effect and external effect And regional competitiveness.

Wuhan robot short board lies in industrial concentration. Although key enterprises represented by Huazhong $\mathrm{CNC}$ strengthen the $\mathrm{R} \& \mathrm{D}$ and production capacity of their own core components, most other enterprises still focus on processing and assembly, lacking key core technologies, and the key core components mainly rely on imports. With the exception of a handful of robotics backbone companies, most robots lack brand recognition. Therefore, Wuhan municipal government should nurture and support a number of leading enterprises to support those enterprises with competitive advantages and development prospects and make them the core of the cluster development of Wuhan robot industry, so as to enhance the overall competitiveness of the robot. Moreover, it is necessary to strengthen cooperation with large foreign companies in technology, draw on their development experience and improve their own production technology. In the industrial agglomeration area, it is necessary to vigorously develop the park economy and guide the robot enterprises to gather in the parks suitable for their existence and development so as to enhance their economic benefits. Based on the characteristics of industries with high relevance and long industrial chain in advanced manufacturing industry, it is necessary to speed up the development of supporting industries for robots, lengthen the industrial chain of robotics and strive to eliminate the bottlenecks in industrial support for the industrialization of robots.

\section{Wuhan Robot to Enhance Business Innovation}

Advanced manufacturing is an important carrier of scientific and technological innovation, the most concentrated and active area for innovation, as well as a fertile ground for innovation and practical application. Wuhan Municipal Government should focus on policies to promote the leapfrog development of advanced manufacturing industry and guide the robot enterprises forward-looking at the forefront of the robot industry at home and abroad, selectively for some R \& $\mathrm{D}$ costs are too high, the research and development cycle is too long, the introduction of The robot technology with obvious benefits is directly introduced and digested, absorbed and innovated, the high grafting of robotics is promoted, the backward advantage is realized, and the leap-forward progress with low cost, short time and high efficiency is achieved.

Adhere to a high starting point to promote innovation in Wuhan robot enterprises leapfrog 
development, based on the existing robotics technology in our country, accelerate the development of high-end robotics industry technology process. The robot enterprises with independent innovation awareness and independent intellectual property rights should be given full support in all aspects so as to enhance the core competitiveness of Wuhan robot enterprises. Constructively build a leading industrial technology $\mathrm{R} \& \mathrm{D}$ system, and strive to promote the collaborative innovation of the main innovations such as production, learning and research to enhance the original innovation capability of enterprises. Finally, it is necessary to promote the application and industrialization of the achievements with core independent intellectual property rights foster the development of strategic emerging industries and enhance the overall innovation level of Wuhan's robot enterprises.

\section{Conclusion}

With the continuous maturation of the field of industrial robots, robots are used more and more widely, making a great contribution to the realization of intelligent and precise manufacturing. Wuhan Industrial Robot has a wide range of application prospects and huge market potential, but also ushered in an unprecedented competitive pressures and challenges. In recent years, our country has made great progress in the research on the robot industry and has made some breakthroughs in key technologies. However, it still lacks breakthroughs in the overall core technology. Although it has been applied in all walks of life, imported robots make up the vast majority. The development of Wuhan robot industry should combine its own characteristics to form its own unique development. Policy support centered on technological progress, technological innovation and enhancing competitiveness can fundamentally solve the impetus and vitality of industrial development and strengthen policy support.

\section{Acknowledgement}

Manufacturing Industry Development Research Center on Wuhan City Circle, Jianghan University

\section{References}

[1] Jun Chen, Yunjun Zhang . Analysis of China's robot industry development policy based on innovation $2.0[\mathrm{~J}]$. (In Chinese)

[2] Wenkui Zhou,Chunli Fu.Study on the Development of Jiangsu Robot Industrial Park [J] .Journal of Cooperative Economy \& Technology, 2017 (09): 34-36.(In Chinese)

[3]Hairong Liu. Tianjin to promote the development of robotics industry [J]. Information Engineering, 2017 (03): 37-38.(In Chinese)

[4] Hui $\mathrm{Xu}$. Kunshan Robot Industry Development Status and Suggestions [J]. China Hi-tech Enterprise, 2016 (27): 3-4.(In Chinese)

[5] Ting Wu. Research on Industrial Robot Industry in Wuhu [D]. Anhui University of Engineering, 2016.(In Chinese)

[6] Shuhua Gao. Reflections on the Fiscal and Tax Policies to Promote the Development of China's Robot Industry [D]. China Academy of Financial Sciences, 2016.(In Chinese)

[7] Minghe Chen. China's robot industry development problems and countermeasures [J]. Science and Technology for Development, 2015 (06): 768-774.(In Chinese)

[8] Bixi Dong. Study on the Forming Mechanism of Industrial Robot Industrial Cluster in Tianjin [D]. Tianjin University of Technology, 2015.(In Chinese)

[9] Hong Liu. Research on the Development Planning of Shanghai Robot Industrial Park [D]. Central South University, 2014.(In Chinese) 


\section{About the author}

Dong Liang ${ }^{1}: 1960$ - a professor at Jianghan University Business School, is mainly engaged in the research of enterprise strategy and government strategy.

Guopei $\mathrm{Cai}^{2}, 1966$ - deputy director of Hubei Institute of Macroeconomics, Master Instructor, the main research directions for macroeconomics and investment economics.

Zhongwei zhang ${ }^{3}: 1994-J i a n g h a n$ University Graduate School of Business, the main research direction for marketing and service engineering. 